\title{
Nutritional Status of Children (Aged 2-5 Years) in Food Secure and Insecure Households in Kuyu Woreda, Oromia, Ethiopia
}

\author{
Tamiru Yazew ${ }^{1 *}$, Agama Daba ${ }^{1}$ \\ Department of Food Science and Nutrition, College of Agriculture, Shambu Campus, Wollega University, \\ Shambu, Ethiopia, Box 38, Shambu, Ethiopia, email: \\ Corresponding Author: tamiruyazew2012@gmail.com
}

\begin{abstract}
Malnutrition, poor child feeding practices and low dietary diversity are common in low income households, where food insecurity is prevalent. Therefore, this study was designed to assess and compare nutritional status of children (aged 2-5 years) in food secure and insecure households of Kuyu woreda. A community based comparative, cross sectional study was conducted in March, 2016. Multi stages sampling methods were employed to select 612 children, out of which 304 children were from food secure and 308 from insecure households. Dietary diversity score was assessed using a $24 \mathrm{hrs}$ recall method. Anthropometric measurements of children were taken and nutritional status was generated using WHO Anthro v.3.2.2. Statistical Package for Social Sciences version 20.0 was used to perform descriptive statistics, independent samples T test and chi-square test analyses. P value $<0.05$ was considered as statistically significant. The prevalence of stunting, underweight and wasting was $48.7 \%, 36.7 \%$ and $20.5 \%$ respectively for children in food insecure households. While the prevalence of stunting was $43 \%$, underweight $30.9 \%$ and wasting $16.8 \%$ for children in food secure households. Daily meal frequency; having breakfast, midmorning, afternoon and bedtime snack among children in food secure households were significantly higher than food insecure households $(\mathrm{p}<0.05)$. In addition, children restriction and pressure during meal were significantly $(\mathrm{p}<0.05)$ higher in food insecure households compared to their counterparts. Moreover, this study found that food secure and insecure households were significantly different in children's dietary diversity scores $\left(x^{2}=13.1, p<0.001\right)$, child feeding practices $\left(x^{2}=11.2, p=0.001\right)$, consumption of dairy products $\left(x^{2}=15.44, p<0.001\right)$ and vitamin A rich fruits and vegetables $\left(x^{2}=8.37, p=0.004\right)$. Most importantly, the study revealed that nutritional statuses of children from food secure households were significantly better compared to those from their counterparts. Therefore, Productivity Safety Net Program with all responsible bodies should be intensified to improve the nutritional status, child feeding practices and dietary diversity scores of children in the study area.
\end{abstract}

Keywords: under nutrition, children aged 2-5 years, feeding practices, food insecurity

DOI: $10.7176 /$ ALST/81-02

Publication date: August $31^{\text {st }} 2020$

\section{Introduction}

Malnutrition in children remains one of the most important public health and developmental problems in the developing world, mainly affecting the poor and under privileged (Poel et al., 2008). Children are most vulnerable to malnutrition in developing countries because of low dietary intakes, lack of appropriate feeding practices, and inequitable distribution of food within the households (Yalew, 2014).

Globally, in 2011, nearly one in four children under-five years of age (165 million or 26\% were stunted, 101 million or $16 \%$ underweight and 52 million children under five years were moderately or severely wasted (UNICEF, 2013). Reducing malnutrition among children under the age of five remains a huge challenge in developing countries of the world. An estimated 230 million under-five children are believed to be chronically malnourished in developing countries (Poel et al., 2008)

Sub Saharan Africa and South Asia are home to three fourths of the world's stunted children. In Sub Saharan Africa (SSA), 40\% of children under five years old were stunted; in South Asia, 39\% were stunted (UNICEF, 2013). In 2012, it also reported that the prevalence of underweight (33\%) and wasting (16\%) is highest in South Asia followed by SSA, which is $21 \%$ and $9 \%$, respectively. 
Nationally, according to the latest Ethiopia mini demographic and health survey (EMDHS) reports, the prevalence of stunting, underweight and wasting in under five children was $40 \%, 25 \%$ and $9 \%$, respectively (CSA, 2014). It is currently the wide epidemic in different regions of Ethiopia.

Although food security is essential to ensure adequate nutrition and prevent hunger, the concepts of food security, optimal nutrition and lack of hunger and under nutrition are interlinked (Ghattas, 2014). Household food insecurity is a critical variable for understanding the nutritional status of children in low income countries; as low income is associated with poor diet, food insecurity, and poor child health (Ali et al., 2013).

Appropriate feeding practices have also been one of the most important for children under five years to tackle child's malnutrition (UNICEF, 2014). The nutrition, growth and development of under five children depend not only on sufficient food, but also on good child feeding and care practices (Raphael et al., 2008). However, a household's capacity to provide feeding and care is dependent on the availability of resources or the absence of constraints within the household (UNICEF, 2013).

In Ethiopia, magnitude of both under nutrition and food insecurity is very high in rural areas where livelihood depends on backward farming system and, Productivity Safety Net Program (PSNP) (Sarah et al., 2013). The nutritional consequences of food insecurity experience include underweight, stunting and wasting, depending on a broad range of contextual, economic and socio cultural factors (Ghattas, 2014).

According to a study done in Tigray region, Ethiopia, the prevalence of stunting, underweight and wasting in food insecure households was high and $52.1 \%, 20.5 \%$ and $12.6 \%$, respectively, while in food secure households was $46.1 \%, 18.1 \%$ and $7.1 \%$ for the above three indices (kahsay et al., 2015). Another study done in Sri Lanka showed that the prevalence of stunting, underweight and wasting was statistically higher among children in food insecure households than food secure ones (Kandeepan et al., 2016).

Appropriate and adequate feeding is a pre-requisite for good nutritional status in any a given time of human life WHO, 2010). In many developing countries, poor quality diets are the major causes attributed to childhood malnutrition (Christina, 2011). A study done in Gojjam, Ethiopia found that food insecurity, dietary diversity and child's meal frequency were the significant determinants of under nutrition in children (Motbainor et al., 2015). Another study done also showed that children who had poor feeding practices had low weight for age, and high nutritional deficiencies (Mehta et al., 2013).

Although Oromia is among the regions producing adequate foods in Ethiopia, it is reported that there is high prevalence rate of child under nutrition compared to prevalence in less productive regions (Hirvonen \& Hoddonott, 2014). The Ethiopian Mini Demographic and Health Survey reported that the prevalence of children stunted, underweight and wasted was $37.5 \%, 22.2 \%$ and $6.9 \%$, respectively in Oromia region (CSA, 2014). However, in rural communities, the prevalence of stunting among children in food insecure households was very high $(61 \%)$ (Abdurahman et al., 2016).

Productive Safety Net Program (PSNP) is an intensive and innovative strategy run by Ethiopian government to respond to chronic food insecurity problems in a systematic manner (Rachel et al., 2006). The program targeted poor and food insecure households (Sarah et al., 2013). Kuyu Woreda is one of the PSNP Woredas and is classified as chronic food insecure as most of the kebeles in the Woreda have been recipients of food aid for more than ten years and till now (PSNP, 2014). The PSNP has also classified households in Kuyu Woreda as 'food secure' if they were graduated and stayed for more than one year being out of the PSNP's aid and 'food insecure' if the households are not graduated and continue relying on the assistances from the PSNP in the form of cash or food aid (KWO, 2015).

Recognizing this, Ethiopia is striving forward to ensure food security and become food self-sufficient as food security is one of the pillars of improved nutrition status. Nevertheless, it does not necessarily mean that food secure households are nutritionally secure; as malnutrition is common in many food secure households (Ghattas, 2014). Food insecurity might further compound nutritional problems because of food shortage leading to inadequate food intake and dietary diversity. Hence, it is plausible to answer research questions such as "Is there a difference in nutritional status of children from food-secure and insecure households?" 
Little is known about how malnutrition, feeding practices and dietary diversity score differ between children in rural communities of food secure and insecure households. Besides, there was no study that compared nutritional status, feeding practices and dietary diversity score of children in food secure and food insecure households of Kuyu Woreda based on the PSNP criteria. The EMDHS was done based on a small sample and thus, does not sufficiently represent the local context of the study Woreda. Therefore, this study was designed to assess nutritional status of children (aged 2-5 years) in food secure and insecure households in Kuyu Woreda, Oromia, Ethiopia.

\section{Materials and Methods}

\subsection{Description of the Study Area}

The study area, Kuyu Woreda is located $156 \mathrm{kms}$ north, Addis Ababa, the capital city of Ethiopia and $42 \mathrm{kms}$ from Fitche town in north Shewa Administrative Zone. Astronomically, it is located at about 9 $9^{\circ} 36^{\prime} 34^{\prime \prime}-$ $9^{\circ} 56^{\prime} 56^{\prime \prime} \mathrm{N}$ latitude and 38 $05^{\prime} 00^{\prime \prime}-38034^{\prime} 13^{\prime \prime E}$ longitude. In Kuyu Woreda, there are a total of three urban and 23 rural kebeles. The numbers of households in the woreda, as registered by the kebele offices in 2007 are 157,004. The rural population of the woreda constitutes 105,046 (67\%) of which $49.79 \%$ are males and the remaining $50.21 \%$ are females. There are 25,796 children under five years out of which 11,239 are children 2 to 5 years old. About 5,237 and 6002 children aged 2-5 years live in food insecure and secure households, respectively. As most parts of Ethiopia's plateaus, Kuyu Woreda receives its maximum annual rainfalls of 1800mm during summer season (June, July and August) (GIGCO, 2014). Likewise, the rainfall for the study area is heavily concentrated in four months (June, July, August and September). The major crops grown in the woreda are teff, wheat, barley, bean and Nug (GIGCO, 2014).

\subsection{Study Design and Period}

A community based comparative cross sectional study was conducted in Kuyu woreda, North Shewa zone, Oromia, Ethiopia. The study was carried out in March 2016.

\subsection{Source Population}

All children aged 2-5 years and live in food secure and insecure households of Kuyu woreda were the source population.

\subsection{Study Population}

All children aged 2-5 years who were randomly selected from food secure and insecure households of four selected kebeles (Sombo Cheka, Halilu Cheri, Dubena Agalo and Wuyye Gose) constituted the study population.

\subsection{Inclusion and Exclusion Criteria}

Children aged 2-5 years and mothers/caregivers who were the residents of the study area at least for 6 months were included from both food secure and insecure households.

Children aged 2-5 years who had deformities and children and mothers/caregivers who were not found at home during three visits were excluded from the study.

\subsection{Sample Size Determination}

For each specific objective, a two-population proportions formula, assumptions and Epi Info version 7.0.8.3 statistical software and $15 \%$ a meaningful expected difference between prevalence of stunting among children in food secure and insecure households, $\mathrm{P} 1=37.5 \%$, and $\mathrm{P} 2=22.5 \%$ [4], were used to estimate the sample size. Among the calculated sample sizes for the three specific objectives (392, 296 and 318), the largest sample size $(n=392)$ with $5 \%$ non-response rate and design effect of 1.5 resulted in sample size, $n=618$ children (309 in each group) was taken as size of the study.

\subsection{Sampling Procedures}

Kuyu woreda was purposively selected as study site since more than half of the households in the woreda receive aids (cash and food items) from PSNP. This was because of shortage of food the households experienced 
following the scarcity of rainfall that resulted in less crop production. There were twenty kebeles which were involved in PSNP to receive aids. Therefore, multstages sampling methods were used to draw samples for the study.

Firstly, from the twenty kebeles of the woreda, four rural kebeles (Sombo Cheka, Halilu Cheri, Dubena Agalo and Wuyye Gose) were randomly selected. Then, all the households in the four kebeles were stratified into food secure and insecure households using the list of households available at the PSNP. The total number of children (aged 2-5 years) months in food secure and insecure households of the four kebeles (Sombo Cheka, Halilu Cheri, Dubena Agalo and Wuyye Gose) were 448, 712, 775 and 865, respectively (KWO, 2015).

Next, the number of children to be selected form food secure and insecure households in each kebele was determined by proportional allocation on the bases of total number of children (aged 2-5 years) in the respective kebeles. Finally, the children were randomly selected from each kebele using list of households in each category. A lottery method was used to select a child for mothers who had more than one children (aged 2-5 years) in a single household.

\subsection{Data Collection Procedures}

The quantitative data were collected using a structured questionnaire adapted from different relevant studies. The questionnaire was first developed in English and then translated into Afan Oromo with some modification from the relevant sources. It was translated back to English by different language experts to check for consistency and then back to Afan Oromo to make interview with local respondents. A pre-tested on 5\% of the sample was done in a kebele not included in the sample to check for its understand-ability, time required completing the questionnaire, etc before the actual data collection began. Based on feedback from the pre-test, necessary corrections and editions were made in terminologies and formatting of the questionnaire. After a training had been given for two days, the data were collected by four nurses (diploma holders) and supervised by one public health professional and the researcher. At the end of each day, the completeness of questionnaires were checked by the principal investigator.

\subsection{Socio-demographic Data}

The socio-demographic variables such as age of the child and mothers, sex of child, head of the household, family size, occupation, education status, and wealth index were collected.

\subsection{Anthropometric Data}

The weight of the child was measured using electronic digital weight scale (to the nearest $0.1 \mathrm{~kg}$ ) with minimum/lightly/clothing and no shoes. Calibration was done before weighing every child by setting it to zero using $5 \mathrm{~kg}$ of known iron metal. The height of the child was also measured without shoes (to the nearest $0.1 \mathrm{~cm}$ ) by using a vertical wooden height board. This was done by placing the child on the measuring board, and standing upright in the middle of board, and then the child's head, shoulders, buttocks, knees and heels touching the board. The age of the child was also calculated in months from their birth date to the day of data collection using a local calendar of event and using information obtained from health extension workers.

\subsection{Children's Dietary Diversity}

The Diet Diversity Score (DDS) and a 24-hour recall method were conducted with mothers regarding their child's intake. Mothers/ caregivers were requested to list all the foods consumed by the child both at home and out of the home in the 24 hours preceding the interview. The seven food groups namely grains, roots, tubers; legumes, nuts, seed; dairy products, flesh foods (meats, fish, poultry); eggs, other fruits and vegetables and vitamin A rich fruits and vegetables were used in this study (WHO, 2010). However, oils and fats were not considered and calculated for DDS; because these foods don't add nutritional quality of the diets. Considering four food groups as the minimum acceptable dietary diversity, a child with a DDS of $<4$, was classified as poor dietary diversity and high if a child DDS was $\geq 4$. 


\subsection{Child Feeding Practices}

An interviewer administered questionnaires concerning feeding practices of the child. Mothers/caregivers were requested to response child's meal per/day, when the child eats meal, food child dislike, breakfast, midmorning, afternoon and bedtime snack, leftover food, pressured, restricted to eat meal. Finally, it was grouped into: good feeding practices if mean score was grater or equal to mean $(\geq 4)$ and poor if mean score was less than mean $(<4)$.

\subsection{Variables of the Study}

The dependent variables were height -for- age (stunting), weight -for -age (underweight) and weight -forheight (wasting) while independent variables were child dietary diversity score and feeding practices, sociodemographic, economic factors and household's food security status.

\subsection{Data Analysis}

Data were edited, cleaned, coded, entered and analysed using SPSS for windows version 20.0. A descriptive statistical analysis was conducted for all quantitative variables to check for outliers, consistency of data and missing values. Height for age, weight for age and weight for height of children were generated from WHO growth standards using WHO Anthro program, version 3.2.2. A child was considered as stunted, underweight and wasted if Z score was below - 2 for each index. Normality of continuous variables was checked using Q-Q plots and Histogram. Independent sample $\mathrm{T}$ test for continuous variables and chi square test for categorical variables were used to find out any significant differences between the children lived in food secure and insecure households. P-value less than 0.05 was considered as statistically significant.

\subsection{Ethical Considerations}

Ethical clearance was obtained from the Institutional Review Board of the College of Medicine and Health Sciences of Hawassa University. After explaining the study procedures for the study subjects, verbal consents were obtained from each mother/caregiver.

\section{Results}

\subsection{Socio-demographic Characteristics}

This study included a total of 612 households of which 304 were food secure and 308 were food insecure households with an overall response rate of $99 \%$. In this study, six households were excluded from the study due to mothers were not found at home during the survey. About $154(50.7 \%)$ and $(48.7 \%)$ of mothers were unable to read and write in food secure and insecure households, respectively.The finding of this study also showed that there was statistically significant difference in the wealth status of children in food secure and insecure households $(\mathrm{p}<.05)$. The mean of child age was 43.52. \pm 10.7 in food secure households and $43.58 \pm 10.36$ in food insecure ones (Table 1). 
Table. 1 Socio-demographic characteristics of the study participants, Kuyu Woreda (N=612), 2016.

\begin{tabular}{|c|c|c|c|}
\hline Variables & FS HHs $(n=304) \quad(\%)$ & FI HHs $(n=308)(\%)$ & P value \\
\hline $\begin{array}{l}\text { Mean ( } \pm \text { SD) age of the mothers } \\
\text { (years) }\end{array}$ & $33.56 \pm 6.867$ & $33.14 \pm 6.78$ & 0.628 \\
\hline Head of households & $268(88.2)$ & $266(86.4)$ & \\
\hline Fathers & $36(11.8)$ & $42(13.6)$ & 0.506 \\
\hline \multicolumn{4}{|l|}{ Mothers } \\
\hline \multicolumn{4}{|l|}{ Educational level of mothers } \\
\hline Unable to read and write & $154(50.7)$ & $150(48.7))$ & \\
\hline Able to read and write & $63(20.7)$ & $63(20.3)$ & 0.828 \\
\hline Elementary school and above & $87(28.6)$ & $95(30.8)$ & \\
\hline \multicolumn{4}{|l|}{ Occupational status of mothers } \\
\hline Housewife only & $50(16.4)$ & 41(13.3) & \\
\hline Farmer & $171(56.3)$ & $145(47.1)$ & $0.005 *$ \\
\hline Others (merchants ) & $83(27.6)$ & $122(39.6)$ & \\
\hline \multicolumn{4}{|l|}{ Educational level of father } \\
\hline Unable to read and write & $112(36.8)$ & $122(39.6)$ & \\
\hline Able to read and write & $61(20.1)$ & $73(23.7)$ & 0.127 \\
\hline Elementary school and above & $103(33.9)$ & $81(26.3)$ & \\
\hline \multicolumn{4}{|l|}{ Occupational status of father } \\
\hline Farmer & $189(62.2)$ & $159(51.6)$ & \\
\hline Daily laborer & $27(8.9)$ & $54(17.5)$ & 0.003* \\
\hline $\begin{array}{l}\text { Others } \quad \text { (gov't employee, } \\
\text { merchant) }\end{array}$ & $59(19.4)$ & $63(20.5)$ & \\
\hline Mean $( \pm \mathrm{SD})$ of Family size & $5.47 \pm 1.52$ & $5.28 \pm 1.439$ & 0.067 \\
\hline \multicolumn{4}{|l|}{ Wealth Index } \\
\hline Low & $51(16.8)$ & $72(23.4)$ & \\
\hline Middle & $150(49.3)$ & $176(57.1)$ & $<0.001$ \\
\hline High & $103(33.9)$ & $60(19.5)$ & * \\
\hline $\begin{array}{l}\text { Mean ( } \pm \text { SD) age of the children } \\
\text { (months) }\end{array}$ & $43.52 . \pm 10.7$ & $43.58 \pm 10.36$ & 0.532 \\
\hline
\end{tabular}

* statistically significant difference observed at $\mathrm{p}<0.05$, FS HHs:- Food Secure Households, FI HHs:Food Insecure Households

\subsection{Child Feeding Practices}

This study showed that $131(42.5 \%)$ children in food insecure and $183(60.2 \%)$ children in food secure households had daily meal frequencies of at least 4 which demonstrated a significant statistical difference (p $<0.001)$. Moreover, this finding showed that there was statistically significant difference between children from food secure and insecure households in the frequency of mini meals such as; breakfast $(\mathrm{p}=0.003)$, midmorning snacks $(p<0.001)$, afternoon snacks $(p<0.029)$ and bedtime snacks $(p<0.001)$. Poor child feeding practices were reported both from food secure and insecure households.

Generally, proportion of poor child feeding practices in food insecure households was significantly higher 118 $(38.3 \%)$ than those in food secure households $81(26.6 \%)\left(x^{2}=7.11, p<.008\right)$. The present study showed that 189 $(61.4 \%)$ of children were restricted from snack and dinner meal in food insecure households compared to 125 $(41.1 \%)$ in food secure $(\mathrm{p}<0.001)$. Likewise, $149(48.4 \%)$ versus $111(36.5 \%)(\mathrm{p}<0.001)$ of children were pressured/pressurized to eat dislike foods in food insecure and secure households, respectively. This study also showed that the proportion of children who had no breakfast $(39.3 \%$ vs. $28 \%)$, no midmorning snack $(78.2 \%$ vs.60.9\%), no afternoon snack (52.6\% vs. $43.8 \%)$ and no bedtime snack ( $88 \%$ vs. $77.3 \%)$ in food insecure and secure households, respectively. This indicates that there was statically significant difference among children in food insecure and secure households. However, the proportions of children who consumed leftover foods were not different between food insecure 117(38\%) and secure $106(34.9 \%)$ households ( $\mathrm{p}=.0677)$ (Table 2) 
Table. 2 Feeding practices of the study children in food secure and insecure households Kuyu Woreda $(\mathrm{N}=612)$, 2016.

$\begin{array}{lcccc}\text { Variables } & \begin{array}{c}\text { FS HHs } \\ (n=304)\end{array} & \begin{array}{c}\text { FI HHs } \\ (n=308)(\%)\end{array} & x^{2} & \text { P value }\end{array}$

(\%)

Child's daily meal frequency

$\geq 4$
$\leq 3$

When does the child eat his/her food?

Up on the child demands

When convenient for the mother

Do you restrict child during his/her meal?

$$
\begin{aligned}
& \text { No } \\
& \text { Yes }
\end{aligned}
$$

Do you pressure the child to eat his/her food?

No
Yes

Was there a leftover food at your home in the

$$
\text { past } 24 \text { hrs? }
$$$$
\text { No }
$$

Yes

What did you do when food is leftover in your home?

Give to animal (discarded in garbage)

Give to the child later

Is there any food that the child dislikes?

\begin{tabular}{lcccc} 
No & $207(68.1)$ & $200(64.9)$ & .685 & 0.408 \\
Yes & $97(31.9)$ & $108(35.1)$ & & \\
Ging practices & $76(25)$ & $61(19.8)$ & 7.11 & $\mathbf{0 . 0 0 8 *}$ \\
$\begin{array}{ll}\text { Good } \\
\text { Poor }\end{array}$ & $81(26.6)$ & $118(38.3)$ & & \\
\hline
\end{tabular}

\subsection{Dietary Diversity Scores of Children}

Cereals, roots and tubers were the most common food groups consumed among children in both food insecure $306(99.4 \%)$ and secure 298(98\%) households. The second commonly consumed food included legumes and nuts, which was $250(81.2 \%)$ in food insecure and $260(85.5 \%)$ in food secure households. Low proportion of dairy products was consumed by children in food insecure $(27.6 \%)$ compared to food secure $(42.2 \%)$ households $(\mathrm{p}<0.001)$.

This study also found that there was statistically significant difference in the consumption of dairy and dairy products $(\mathrm{p}<.001)$ and vitamin rich fruits and vegetables $(\mathrm{p}=.004)$ between children from food insecure $(12.3 \%)$ and secure households (21\%). However, no statistically significant difference was observed in the consumption of cereals; grains, roots and tubers, pulses; legumes and nuts, vitamin rich A fruits and vegetables, flesh foods and eggs between children in food insecure and secure households ( $>00.05$ ).

As shown in Table 3, 97 (31.5\%) and 139 (45.7\%) of children in food insecure and secure households had DDS $\geq 4$, respectively with statistically significant difference $\left(\mathrm{x}^{2}=13.1 \mathrm{p}<0.001\right)$. 
Table. 3 Dietary diversity scores of the study children in food secure and insecure households Kuyu Woreda $(\mathrm{N}=612), 2016$.

\begin{tabular}{|c|c|c|c|c|}
\hline \multirow[t]{2}{*}{ Food groups } & FS HHs & \multirow{2}{*}{$\begin{array}{c}\text { FI HHs } \\
(n=308)(\%)\end{array}$} & \multirow[t]{2}{*}{$x^{2}$} & \multirow[t]{2}{*}{ Pvalue } \\
\hline & $(n=304) \quad(\%)$ & & & \\
\hline Cereals/Root/Tubers & $298(98)$ & $306(99.4)$ & 2.08 & 0.15 \\
\hline Legumes/Nuts & $260(85.5)$ & $250(81.2)$ & 2.09 & .0148 \\
\hline Dairy/dairy products & $130(42.2)$ & $85(27.6)$ & 15.44 & $<0.001 *$ \\
\hline Meat/Fish/Poultry & $61(20.1)$ & $52(16.9)$ & 1.029 & 0.31 \\
\hline Eggs & $58(19.1)$ & $46(14.9)$ & 1.86 & 0.172 \\
\hline Other fruits and vegetables & $147(48.8 \%)$ & $130(42.2 \%)$ & 2.33 & 0.127 \\
\hline Vitamin A rich F\&V & $64(21.1)$ & $38(12.3)$ & 8.37 & $0.004 *$ \\
\hline \multicolumn{5}{|l|}{ Dietary diversity scores } \\
\hline High & $139(45.7)$ & $97(31.5)$ & 13.1 & $<0.001 *$ \\
\hline Low & $165(54.3)$ & $211(68.5)$ & & \\
\hline
\end{tabular}

\subsection{Nutritional Status of Children}

The current study demonstrated that the magnitude of malnutrition was significantly higher among children from food insecure households compared to those from food secure households. For instance, stunting was ( $48.7 \%$ vs. $43.1 \%)$, underweight (36.7\% vs. $30.9 \%)$ and wasting $(20.5 \%$ vs. $16.8 \%)$ in food insecure and secure households, respectively with the level of significance in each case to be $\mathrm{p}<0.05$ (Figure 1 ).

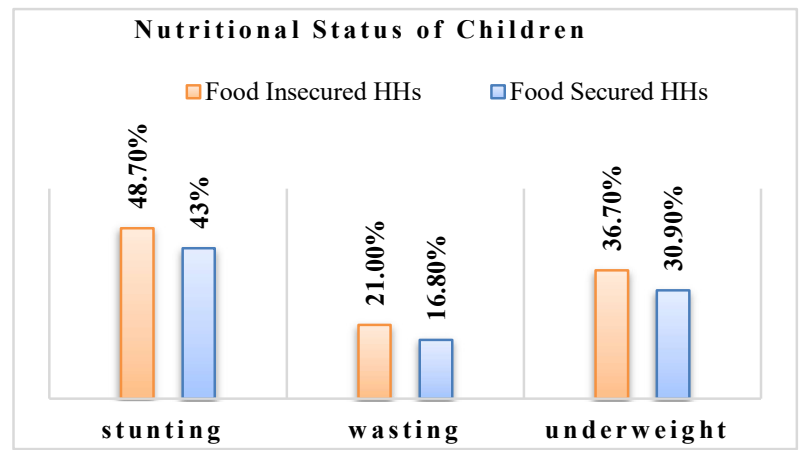

Figure 1. Nutritional status of children in food secure and insecure households Kuyu Woreda (N=612), 2016.

\section{Discussion}

This study provided the opportunity to assess and compare nutritional status, feeding practices and dietary diversity score of children (aged 2-5 years) from food insecure and secure households in Kuyu Woreda. The results from this study showed that there was statistically significant difference in feeding practices and dietary diversity score between children in food insecure and secure households. The prevalence of stunting, underweight and wasting among children from food insecure households was significantly higher compared to children from food secure households $(\mathrm{p}<0.05)$. 
The study revealed that children in food insecure households had low proportion of daily meal $\geq 4$ compared to those in food secure $(\mathrm{p}<0.05)$. This could be due to an indication that being food secure is a way to increase child's daily meal frequencies. Studies conducted in Ethiopia and Zambia showed that the proportion of daily meal $\geq 4$ among children during the survey was high (Disha et al., 2012) compared to child's daily meal in both food insecure and secure households of this study. On contrary, a study done in Ethiopia (Ghate, 2014) reported that proportion of daily meals $\geq 4$ among children during survey was lower compared to both food insecure and secure households of the current study. This could be due to the present survey was conducted in the season of postharvest that may increase food availability, accessibility and utilization by communities at household level.

Regarding child's mini meals, this study showed that high proportion of children from food insecure households did not take breakfast and midmorning snack compared to those from food secure $(p<0.05)$. This situation might be related to economic status of the households. Because of their economic status, food insecure households have limited capacity to regularly provide mini meals for the child. A study conducted in Nigeria also noted that high proportion of children from households with low socioeconomic status did not take breakfast and midmorning snack compared to those from households with better economic status. However, the proportion reported in Nigeria (16\%) (Ntwenya et al.,2015) was much lower compared to both food insecure and secure households of this In the present study, high proportion of restriction and pressure to eat meal were also reported among children in food insecure households than food secure $(\mathrm{p}<0.05)$. Similarly, Ventura \& Birch, 2008; Jansen et al., 2012 reported that high proportion of pressure to eat meal and restriction access to certain foods were observed among children in food insecure households. This might be due to the rate of poverty, the low purchasing power and limited access to the food by households in food insecure.

According to the finding of this study, high proportion of poor feeding practices (fed leftover food) was found in food insecure households than food secure $(\mathrm{p}<0.05)$. This could not be only due to lack of food but also lack of knowledge of mothers on child feeding practices. This is supported by previous studies done in northern Ethiopia (Yalew, 2014) and Sothern Ethiopia (Godana \& Mengistie, 2013), which showed that many children in rural communities are fed on leftover food that are low in quality.

Moreover, this study showed that high consumption of dairy products and vitamin A rich fruits and vegetables were observed among children in food secure households compared to food insecure $(\mathrm{p}<0.05)$. Similarly, Ntwenya et al., 2015 \& Naser et al., 2015, noted that high proportion of dairy products and vitamin rich fruits and vegetables were consumed among children in food secure households than those in food insecure households and difference was statistically significant. Farmers in food secure households could largely on dairy products and home gardening through irrigation to generate household incomes; and this may increase the consumption of these food groups.

The study also revealed that the proportion of children with DDS $\geq 4$ was low among children from food insecure households than among food secure households $(\mathrm{p}<.05)$. This finding was coincided with studies done in Ethiopia (Kahsay et al., 2015) and Malaysia (Naser et al., 2015). Similarly, a study done in Tanzania also noted that the risk for inadequate dietary diversity was higher in food insecure households compared to food secure (Ntwenya et al., 2015). This might be due to the reasons that being food secure is a way to increase dietary diversity score compared to food insecure households.

According to this study, the prevalence of stunting, underweight and wasting among children in food secure households was lower compared to those in food insecure households $(p<0.05)$. This could be due to the signal that indicated being food secure is the way which is important to address nutrition security and lower the prevalence of under nutrition in children under five years. However, compared to regional (Oromia) and national (Ethiopia)(CSA, 2014), the prevalence of under nutrition among children in both comparison groups of this study was higher. This might be due to data collected from the poor communities where food insecurity is prevalent and seasonal variation which may affect the nutritional status of children in the study area.

This study was in line with studies done in Ethiopia (Kahsay et al., 2015) and Sri Lanka (Kandeepan et al.,2016), which found that there was statistically difference in the prevalence of stunting and underweight among children in food insecure and secure households $(\mathrm{p}<.05)$. In contrast, a study done in Ethiopia by (Abdurahman et al., 2016) reported that there was no statistically difference in the prevalence of stunting and underweight between children in food insecure and secure households. 
Concerning the magnitude of wasting, this study confirmed studies done in Ethiopia Abdurahman et al., 2016; Sri Lanka (Kandeepan et al.,2016), which reported that there was statistically significant difference in the prevalence of wasting between children in food insecure and secure households. On contrary, studies done in Tigray (Kahsay et al., 2015), Ghana (Saaka \& Osman, 2015) and Malaysia (Naser et al.,2015) showed that there was no statistically significant difference observed in the prevalence of wasting between children from food insecure and secure households.

\section{Conclusion}

This study demonstrated that there was statistically significant difference in child feeding practices, child's daily meal, mini meals, pressure and restriction of child during meals between food insecure and secure households. Consumption of dairy products, vitamin rich fruits and vegetables and dietary diversity scores were significantly higher among children in food secure households than their counterparts. Furthermore, this study concluded that the magnitude of stunting, underweight and wasting of children in food insecure households was higher compared to those children from food secure households.

\section{Acknowledgements}

The authors acknowledge Hawassa and Wollega Universities, Kuyu Woreda Health and Agriculture offices, data collectors, supervisors and mothers who participated in the study.

\section{References}

Abdurahman, A. et al. (2016). Household Food Insecurity May Predict Underweight and Wasting among Children Aged 24-59 Months, Ecology of Food and Nutrition: 21:18.

Ali, D, et al. (2013). Household Food Insecurity Is Associated with Higher Child Under nutrition in Bangladesh, Ethiopia, and Vietnam, but the Effect Is Not Mediated by Child Dietary Diversity; Journal of Community and International Nutrition, Volume 143:pp 2015-2021.

Christina, A. (2011). Dietary Diversity is Associated with Nutrient Intakes and Nutritional Status of Children in Ghana", Asian Journal of Medical Sciences; Volume 2: 105-109.

CSA. (2014). Ethiopian Mini Demographic and Health Survey, Addis Ababa: Central Statistical Agency 2014

Disha, A., Rawat, AR., Subandoro, A \& Menon, P. (2012). Infant And Young Child Feeding Practices In Ethiopia, Zambia and Their Association With Child Nutrition", Analysis of Demographic and Health Survey Data. African Journal of Food Agriculture, Nutrition and Development, Volume 12 (2): 589-5914.

Ghate, P. (2014). Food Consumption Patterns and Malnourished Ethiopian Children, Is There a Link? Expo International Multidisciplinary Research Journal, 4(2) Section 1, ISSN: 2250 - 1630.

Ghattas, H. (2014).Food Security and Nutrition in the context of the Global Nutrition Transition, Technical Paper. FAO, Rome 2014

GIGCO, (2014). Gabra Guracha Town Environmental condition recording office North Shewa Zone, Ethiopia.

Godana, W \& Mengistie, B. (2013). Determinants of acute diarrhea among children under five years of age in Derashe District, Southern Ethiopia. Journal of Remote and Health Research, Education, Practices, Policy, 13: 2329; ISSN1445-6354.

Hirvonen, A. \& Hoddinott, J. (2014).Targeting food security interventions when "everyone is poor": The case of Ethiopia's Productive Safety Net Programme. International Food Policy Research Institute.

Jansen,WP, et al. (2012). Children's eating behavior, feeding practices of parents and weight problems in early childhood", International Journal of Behavioral Nutrition and Physical Activity, 9:30.

Kahsay, A., Mulugeta, A., \& Seid,O. (2015). Nutritional Status of Children aged 6-59 months from Food Secure and Food Insecure Households in Rural Communities of Saesie Tsaeda-Emba District, Ethiopia", Comparative Study. Clinical Medicine Research, Vol 4(1): pp.51-65.

Kandeepan,k., Balakumara, S., \& Arasaratnama, V. (2016). Nutritional status and food insecurity among the children in Northern Sri Lanka, Procedia Food Science, 6: pp 220-224.

Mehta, H., Groetch, M., \& J. Wang, J. (2013). Growth and Nutritional Concerns in Children with Food Allergy; Curr Opin Allergy Clin Immunol, 13(3): 275-279.

Motbainor, A., Worku, A., \& Kumie, A. (2015). Stunting Is Associated with Food Diversity while Wasting with Food Insecurity among Under five Children in East and West Gojjam Zones of Amhara Region, Ethiopia, PLoS One, Vol 10(8).

Naser,I.A., Jalil, R.A., \& Abdullah,M.R. (2015). Assessment of Food Insecurity and Nutritional Outcomes in Bachok, Kelantan, Journal of Nutrition and food science, 5:3.pp 2155-9600.

Ntwenya, J.E. et al. (2015). Dietary Patterns and Household Food Insecurity in Rural Populations of Kilosa District, Tanzania, PLoS ONE.10(5). 
Ogbimi, G.E \& Ogunba, O.B. (2011). Nutritional Quality of the Lunches of Children in Day Care in Osun State of Nigeria, African Journal of Food, Agriculture, Nutrition and Development, 2011, Volume 11(4); pp 50535063.

Poel, E. et al. (2008). Socioeconomic inequality in malnutrition in developing countries, Vol 86(4): pp 282-291.

PSNP. (2014). Productive Safety Net Program): Ethiopia's Productive Safety Net Program Phase IV Program Implementation, Ministry of Agriculture Addis Ababa.

Rachel, S, et al. (2006). Ethiopia Productive Safety Net Program: Policy, Program, and Institutional Linkages.

Raphael,O.B., Funk,I.O., Segun, B.F., \& E.S. Foluke, E.S.(2011). Prevalence and Determinants of Malnutrition among Under-five Children of Farming Households in Kwara State, Nigeria", Journal of Agricultural Science, vol 3(3): 173-181.

Saaka, M., and Osman,M.S. (2015). Does Household Food Insecurity Affect the Nutritional Status of Preschool Children Aged 6-36 Months?, International Journal of Population Research, 2015Article ID 304169, 12 pages.

Sarah,C.B, et al. (2013). Targeting Food Security Interventions: The Case of Ethiopia's Productive Safety Net Program, International Food Policy Research Institute.

UNICEF. (2013). United Nations Children's Fund and World Health Organization, Joint UNICEF WHO-The World Bank malnutrition database: estimates for 2012 and launch of interactive data dashboards.

UNICEF. (2010). United Nations Children's Fund: Causes and most vulnerable to under nutrition-UNICEF conceptual framework, New York.

UNICEF. (2014). United Nations Children's Fund: Assessment of food security in Ethiopia”, Asian Journal of Agriculture.

Ventura, K.A., \& L. Birch, L. (2008). Does parenting affect children's eating and weight status? International Journal of Behavioral Nutrition and Physical Activity, 2008; 5:15

WHO. (2010). World Health Organization: Indicators for assessing infant and young child feeding practices part 3- country profiles, Geneva, Switzerland, 2010.

WOKW. (2015). Woreda Office of Kuyu Woreda: North shewa Zone, Ethiopia.

Yalew,B.M.(2014). Prevalence of malnutrition and associated factors among children age 6-59 months at Lalibela Town Administration, North Wollo Zone, Anrs, Northern Ethiopia, J Nutr Disorders Ther; Volume 4: 132. 